\title{
Asymmetric $\alpha$-amination of 3-substituted oxindoles using chiral bifunctional phosphine catalysts
}

\author{
Qiao-Wen Jin ${ }^{1}$, Zhuo Chai ${ }^{2}$, You-Ming Huang ${ }^{2}$, Gang Zou ${ }^{* 1, \S}$ and Gang Zhao ${ }^{* 2, \pi}$
}

\author{
Full Research Paper \\ Address: \\ ${ }^{1}$ Laboratory of Advanced Materials and Institute of Fine Chemicals, \\ East China University of Science and Technology, 130 Meilong Road, \\ Shanghai 200237, People's Republic of China and ${ }^{2}$ Key Laboratory of \\ Synthetic Chemistry of Natural Substances, Shanghai Institute of \\ Organic Chemistry, Chinese Academy of Sciences, 345 Lingling \\ Road, Shanghai 200032, People's Republic of China \\ Email: \\ Gang Zou* - zougang@ecust.edu.cn; Gang Zhao* - \\ zhaog@mail.sioc.ac.cn \\ * Corresponding author \\ $\S$ Fax: (+86)-21-6425-3881 \\ I Fax: (+86)-21-6416-6128 \\ Keywords: \\ 3-aminooxindoles; asymmetric catalysis; phosphine catalyst; \\ tetrasubstituted stereogenic carbon centers
}

Beilstein J. Org. Chem. 2016, 12, 725-731.

doi:10.3762/bjoc. 12.72

Received: 29 November 2015

Accepted: 05 April 2016

Published: 15 April 2016

This article is part of the Thematic Series "Bifunctional catalysis".

Guest Editor: D. J. Dixon

(c) 2016 Jin et al; licensee Beilstein-Institut.

License and terms: see end of document.

\begin{abstract}
A highly enantioselective $\alpha$-amination of 3 -substituted oxindoles with azodicarboxylates catalyzed by amino acids-derived chiral phosphine catalysts is reported. The corresponding products containing a tetrasubstituted carbon center attached to a nitrogen atom at the $\mathrm{C}-3$ position of the oxindole were obtained in high yields and with up to $98 \%$ ee.
\end{abstract}

\section{Introduction}

Recently, chiral 3-substituted oxindoles have been attractive targets in asymmetric synthesis due to their abundance in the structures of numerous natural products and pharmaceutically active compounds [1]. In particular, the chiral 3-aminooxindoles containing a tetrasubstituted carbon center have been recognized as core building blocks for the preparation of many biologically active and therapeutic compounds [2-7]. As a type of commercially available electrophilic amination reagents, azodicarboxylates have been extensively used in both asymmetric organocatalysis and metal catalysis for the construction of this type of structures. For example, Chen et al. reported the first organocatalytic enantioselective amination reaction of 2-oxindoles catalyzed by biscinchona alkaloid catalysts [8]. Zhou $[9,10]$ and Barbas $[11,12]$, have independently reported similar organocatalytic processes. In the field of metal catalysis, Shibasaki et al. reported the reaction between C3-substituted oxindole and azodicarboxylates, using homodinuclear or monometallic Ni-Schiff base complexes as catalysts [13]; Feng et al. also developed a similar procedure with chiral $N, N^{\prime}-$ dioxide-Sc(III) complexes as catalysts [14]. Despite these 
impressive advances, current catalytic systems still more or less suffer from limitations such as long reaction times, relatively large catalyst loading in most organocatalytic systems and in some cases unsatisfactory yields and/or enantioselectivities. Therefore, the development of more efficient catalytic systems for the asymmetric $\alpha$-amination of 3 -substituted oxindoles with azodicarboxylates is still desirable.

Chiral organophosphine catalysis [15-18] has captured considerable attention over the past decades owing to its high catalytic efficiency in a variety of reactions such as azaMorita-Baylis-Hillman reactions [19-21], Rauhut-Currier reactions [22-27], Michael addition reactions [28-35], and various cycloadditions [36-39]. In recent years, our group has focused on the development of novel amino acid-derived chiral bifunctional organophosphine catalysts, which have successfully applied to catalyze various asymmetric reactions [40,41]. As a general concept, a tertiary phosphine adds to an electrophilic reactant to form a zwitterion which serves as either a nucleophile or a Bronsted base to participate in the catalytic cycle. In 2015 , we reported a novel asymmetric dual-reagent catalysis strategy based on these chiral phosphine catalysts [42], in which the zwitterion in situ generated from the chiral phosphine and methyl acrylate acted as an efficient catalyst for the asymmetric Mannich-type reaction. As an extension of this work, we then wondered if other electrophilic partners instead of methyl acrylate could be used to generate similar catalytically active species in situ. Also inspired by the Mitsunobu reaction [43], we reported herein the reaction of azodicarboxylates with 3-substituted oxindole catalyzed by chiral amino acid-derived organophosphine catalysts, in which the zwitterions in situ generated from the phosphine and azodicarboxylates serve as highly efficient catalysts [44] (Scheme 1).

\section{Results and Discussion}

Initially, the reaction between 3-phenyloxindole 1a and DEAD (diethyl azodicarboxylate, 2a) was selected as the model reaction for the evaluation of chiral phosphine catalysts (Table 1). Using bifunctional thiourea catalysts $\mathbf{4 a}$ and $\mathbf{4 b}$, the reaction proceeded smoothly at room temperature to afford the product 3a in good yields, albeit with low enantiomeric excesses (ee) (Table 1, entries 1 and 2). When the thiourea moiety in the catalysts were replaced by amides, the enantioselectivities were greatly improved (Table 1, entries 3-6). The examination of

Mitsunobu reaction :

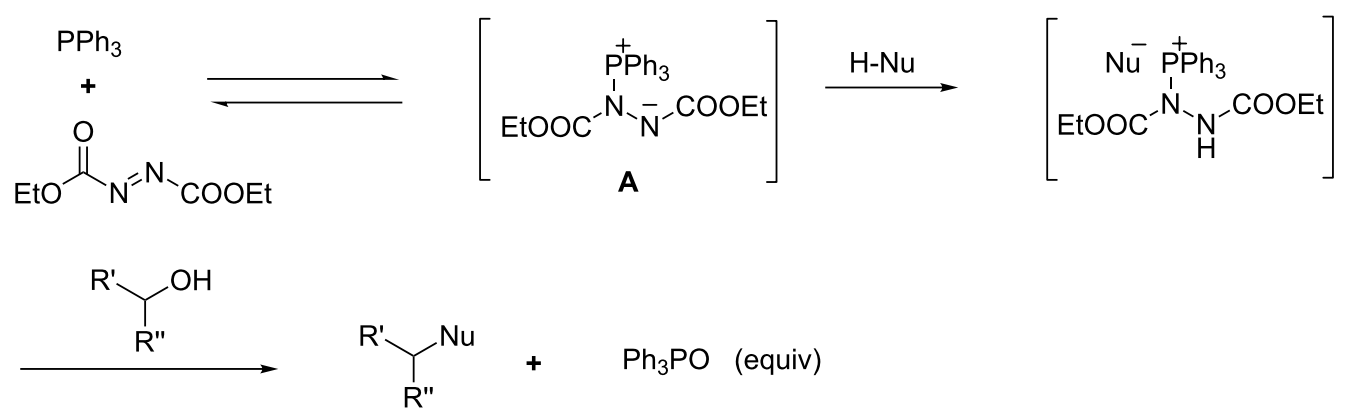

This work :

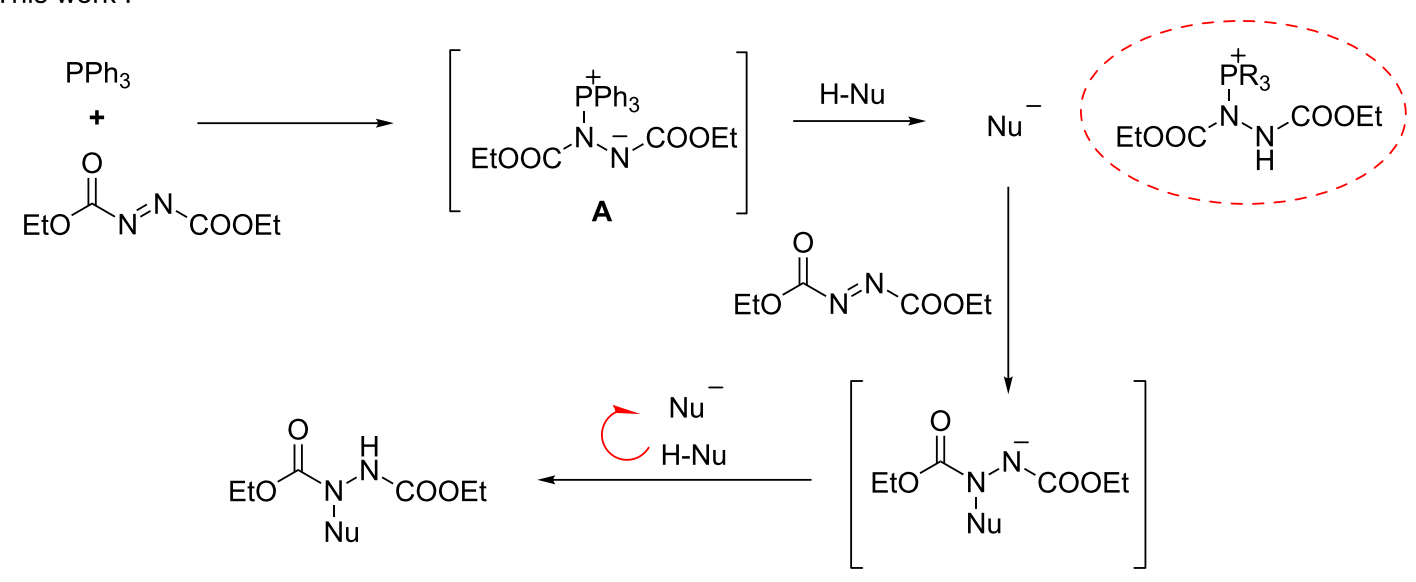


Table 1: Catalyst screening.

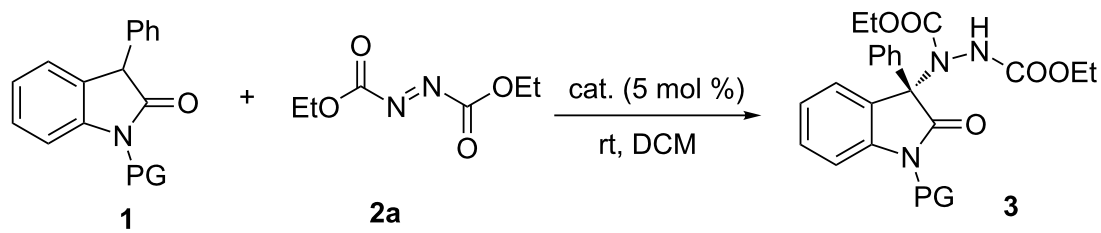<smiles>CC[C@H](C)C(Cc1ccccc1)NC(=S)Nc1cc(C(F)(F)F)cc(C(F)(F)F)c1</smiles>

$4 a$<smiles>CC(C)(C)[C@H](CPc1ccccc1)NC(=S)Nc1cc(C(F)(F)F)cc(C(F)(F)F)c1</smiles>

4b<smiles>CC(C)(C)[C@H](CPc1ccccc1)NC(=O)c1cc(C(F)(F)F)cc(C(F)(F)F)c1</smiles><smiles>[R]c1ccccc1C(=O)NC(Cc1ccccc1)Cc1ccccc1</smiles>

4d $\mathrm{R}=3,5-\mathrm{CF}_{3}$

$4 \mathrm{e} R=\mathrm{H}$

4f $\mathrm{R}=4-\mathrm{NO}_{2}$

4c

\begin{tabular}{|c|c|c|c|c|c|}
\hline Entry ${ }^{a}$ & $P G$ & Catalyst & $t(\min )$ & Yield $(\%)^{b}$ & ee $(\%)^{c}$ \\
\hline 1 & $\operatorname{Boc}(1 a)$ & $4 a$ & 5 & 72 & 17 \\
\hline 2 & $\operatorname{Boc}(\mathbf{1 a})$ & $4 b$ & 5 & 79 & 39 \\
\hline 3 & $\operatorname{Boc}(1 a)$ & $4 c$ & 5 & 89 & 61 \\
\hline 4 & $\operatorname{Boc}(\mathbf{1 a})$ & $4 d$ & 5 & 85 & 83 \\
\hline 5 & $\operatorname{Boc}(1 a)$ & $4 e$ & 5 & 88 & 60 \\
\hline 6 & $\operatorname{Boc}(1 a)$ & $4 f$ & 5 & 70 & 64 \\
\hline 7 & $H(\mathbf{1 b})$ & $4 d$ & 40 & 66 & 17 \\
\hline 8 & $\mathrm{Bn}(1 \mathrm{c})$ & $4 d$ & 60 & 50 & 0 \\
\hline
\end{tabular}

${ }^{\mathrm{a}} 0.1 \mathrm{mmol}$ scale in $1.0 \mathrm{~mL}$ of DCM. ${ }^{\mathrm{b}}$ Isolated yield. ${ }^{\mathrm{C}}$ Determined by chiral HPLC analysis.

catalysts $\mathbf{4 c - f}$ with further fine-tuning on the acyl group revealed $\mathbf{4 d}$ as the optimal catalyst for this transformation (Table 1, entry 4). Different from $N$-Boc-oxindole, using $N$-unprotected oxindole and $N$-benzyl-substituted oxindole as the substrates, accomplished the reaction with every low enantioselectivity (Table 1 , entries 7 and 8 ), incidated the $N$-Boc protecting group is crucial for this system.

Next, the influence of solvents and reaction temperature on the reaction were investigated with the best catalyst (Table 2 ). The use of both polar solvents (ethyl ether, tetrahydrofuran, acetone, acetonitrile or ethyl alcohol) including other chlorinated solvents such as chloroform, 1,2-dichloroethane and 1,1,2trichloroethane or the less polar solvent toluene gave no improvement in enantioselectivity in comparison to the originally used DCM (Table 2, entries 1-9). To our delight, lowering the reaction temperature increased the reaction yield significantly (Table 2, entries 10-16), while the highest ee value (90\%) with DEAD was obtained at $-30{ }^{\circ} \mathrm{C}$ (Table 2, entry 13 ). Interestingly, the use of other azodicarboxylates with larger $\mathrm{R}$ group as amination reagent revealed different optimum reaction temperatures for the best enantioselectivity, and $-78^{\circ} \mathrm{C}$ was identi- fied as optimal for di-tert-butyl azodicarboxylate (2d, DBAD, $93 \%$ ee, Table 2, entry 20). It's worth mentioning that the reaction could still proceed to completion within 5 minutes under such low reaction temperatures.

With the optimized reaction conditions in hand, a variety of oxindoles 1 and azodicarboxylates 2 were then examined to probe the scope of the reaction (Table 3). In general, the catalytic system showed excellent efficiency for all the substrates examined to provide good to excellent yields and enantioselectivities within a very short reaction time $(5 \mathrm{~min})$. The use of the sterically more hindered DBAD is much more favored than DEAD in terms of enantioselectivity. The substitution type including different electronic nature and/or positions of the substituents on the benzene ring of the oxoindole skeleton or 3-aryl group showed no pronounced influence on the reaction in terms of both yield and enantioselectivity. It is noteworthy that products $\mathbf{3 i}, \mathbf{3 q}, \mathbf{3 r}$ and $\mathbf{3 s}$, which contain a fluorine atom, were obtained in good yield with good to execellent ee (Table 3, entries 6 and 14-16). Enantiomerically enriched fluorine-containing 2-oxoindoles are of great significance in drug discovery and development [45]. Unfortunately, there was no ee observed when 
Table 2: Optimization of conditions.

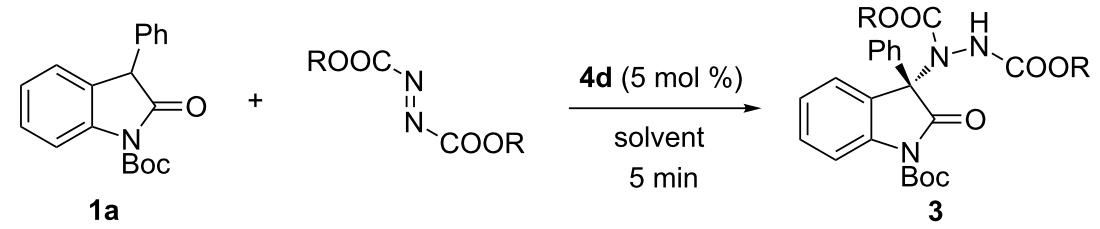

\begin{tabular}{|c|c|c|c|c|c|}
\hline Entry ${ }^{a}$ & $\mathrm{R}$ & Solvent & $T\left({ }^{\circ} \mathrm{C}\right)$ & Yield $(\%)^{\mathrm{b}}$ & ee $(\%)^{c}$ \\
\hline 1 & Et & $\mathrm{Et}_{2} \mathrm{O}$ & $\mathrm{rt}$ & $64(3 a)$ & 65 \\
\hline 2 & Et & THF & $\mathrm{rt}$ & $62(3 a)$ & 39 \\
\hline 3 & Et & acetone & $\mathrm{rt}$ & $62(3 a)$ & 35 \\
\hline 4 & Et & acetonitrile & $\mathrm{rt}$ & $41(3 a)$ & 5 \\
\hline 5 & Et & $\mathrm{EtOH}$ & $\mathrm{rt}$ & $78(3 a)$ & 0 \\
\hline 6 & Et & toluene & $\mathrm{rt}$ & $70(3 a)$ & 79 \\
\hline 7 & Et & $\mathrm{CHCl}_{3}$ & $\mathrm{rt}$ & $66(3 a)$ & 79 \\
\hline 8 & Et & 1,2-dichloroethane & $\mathrm{rt}$ & $66(3 a)$ & 77 \\
\hline 9 & Et & 1,1,2-trichloroethane & $\mathrm{rt}$ & $74(3 a)$ & 53 \\
\hline 10 & Et & $\mathrm{DCM}$ & 0 & $81(3 a)$ & 83 \\
\hline 11 & Et & $\mathrm{DCM}$ & -10 & $93(3 a)$ & 84 \\
\hline 12 & Et & $\mathrm{DCM}$ & -20 & $86(3 a)$ & 85 \\
\hline 13 & Et & DCM & -30 & $87(3 a)$ & 90 \\
\hline 14 & Et & $\mathrm{DCM}$ & -40 & $87(3 a)$ & 84 \\
\hline 15 & Et & $\mathrm{DCM}$ & -50 & $93(3 a)$ & 81 \\
\hline 16 & Et & $\mathrm{DCM}$ & -78 & $85(3 \mathbf{a})$ & 68 \\
\hline 17 & $\mathrm{iPr}$ & $\mathrm{DCM}$ & -30 & $95(3 b)$ & 82 \\
\hline 18 & $\mathrm{iPr}$ & $\mathrm{DCM}$ & -78 & $93(3 b)$ & 89 \\
\hline 19 & $t-\mathrm{Bu}$ & $\mathrm{DCM}$ & -30 & $87(3 d)$ & 64 \\
\hline 20 & $t-\mathrm{Bu}$ & $\mathrm{DCM}$ & -78 & $80(3 d)$ & 93 \\
\hline
\end{tabular}

${ }^{\mathrm{a}} 0.1 \mathrm{mmol}$ scale in $1.0 \mathrm{~mL}$ of solvent. ${ }^{\mathrm{b}}$ Isolated yield. ${ }^{\mathrm{C}}$ Determined by chiral HPLC analysis.

Table 3: Substrate scope.

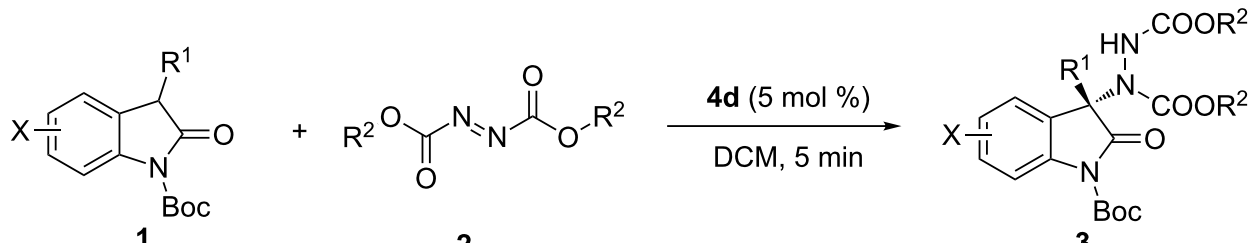

\begin{tabular}{|c|c|c|c|c|c|}
\hline Entry ${ }^{a}$ & $x$ & $\mathrm{R}^{1}$ & $\mathrm{R}^{2}$ & Yield(\%) & $\mathrm{ee}(\%)^{c}$ \\
\hline 1 & $\mathrm{H}$ & $\mathrm{Ph}$ & $t-\mathrm{Bu}$ & $87(3 d)$ & 93 \\
\hline 2 & 5-Me & $\mathrm{Ph}$ & $t$-Bu & $85(3 e)$ & 96 \\
\hline 3 & 5-OMe & $\mathrm{Ph}$ & $t$-Bu & $88(3 f)$ & 96 \\
\hline 4 & 5-Me & $\mathrm{Ph}$ & Et & $88(\mathbf{3 g})$ & 86 \\
\hline 5 & 5-OMe & $\mathrm{Ph}$ & Et & $84(3 h)$ & 88 \\
\hline 6 & $5-\mathrm{F}$ & $\mathrm{Ph}$ & Et & $84(\mathbf{3 i})$ & 87 \\
\hline 7 & $5-\mathrm{Cl}$ & $\mathrm{Ph}$ & Et & $85(\mathbf{3} \mathbf{j})$ & 90 \\
\hline 8 & $6-\mathrm{Cl}$ & $\mathrm{Ph}$ & Et & $87(3 k)$ & 87 \\
\hline 9 & $\mathrm{H}$ & 4- $\mathrm{MeC}_{6} \mathrm{H}_{4}$ & $t$-Bu & $89(3 \mathrm{I})$ & 81 \\
\hline 10 & $\mathrm{H}$ & $4-\mathrm{OMeC}_{6} \mathrm{H}_{4}$ & $t$-Bu & $85(3 m)$ & 95 \\
\hline
\end{tabular}




\begin{tabular}{|c|c|c|c|c|c|}
\hline 11 & $\mathrm{H}$ & $4-\mathrm{MeC}_{6} \mathrm{H}_{4}$ & Et & $90(3 n)$ & 81 \\
\hline 12 & $\mathrm{H}$ & $4-t-\mathrm{BuC}_{6} \mathrm{H}_{4}$ & Et & 82 (3o) & 87 \\
\hline 13 & $\mathrm{H}$ & $3-\mathrm{OMeC}_{6} \mathrm{H}_{4}$ & Et & $86(3 p)$ & 87 \\
\hline 14 & $\mathrm{H}$ & $4-\mathrm{FC}_{6} \mathrm{H}_{4}$ & $t-\mathrm{Bu}$ & $87(3 q)$ & 95 \\
\hline 15 & $\mathrm{H}$ & $4-\mathrm{FC}_{6} \mathrm{H}_{4}$ & Et & $89(3 r)$ & 85 \\
\hline 16 & 5-Me & $4-\mathrm{FC}_{6} \mathrm{H}_{4}$ & $t-\mathrm{Bu}$ & 85 (3s) & 98 \\
\hline 17 & $5-\mathrm{Me}$ & $4-\mathrm{MeC}_{6} \mathrm{H}_{4}$ & $t$-Bu & $86(3 t)$ & 96 \\
\hline 18 & $\mathrm{H}$ & $\mathrm{Me}$ & Et & $72(3 u)$ & 0 \\
\hline
\end{tabular}

${ }^{\mathrm{a}} 0.1 \mathrm{mmol}$ scale in $1.0 \mathrm{~mL}$ of $\mathrm{DCM}$. At $-78^{\circ} \mathrm{C}$ when $\mathrm{R}=t-\mathrm{Bu}$, at $-30^{\circ} \mathrm{C}$ when $\mathrm{R}=\mathrm{Et}$. ${ }^{\mathrm{b}}$ Isolated yield. ${ }^{\mathrm{C}}$ Determined by chiral $\mathrm{HPLC}$ analysis.

the 3-substituent was changed to an alkyl group (Table 3, entry $18)$.

Subsequently, a scale-up experiment on $1.0 \mathrm{mmol}$ scale of the reaction was examined, and the corresponding product could be obtained smoothly with a slightly reduced yield $(70 \%)$ and ee $(85 \%)$. The ee value of the product could be raised to $96 \%$ after a single recrystallization step (Scheme 2 ). The product could be deprotected to provide the known compound 5 with no deterioration in enantioselectivity. The absolute configuration of $\mathbf{3 a}$ was deduced to be $S$ by comparison the specific optical rotation data of 5 with literature data [10,12], and the absolute configurations of other adducts $\mathbf{3 b}-\mathbf{t}$ were assigned by analogy.

To get some insight into this reaction, ${ }^{31} \mathrm{P}$ NMR of the mixture of $4 \mathbf{d}(0.5 \mathrm{~mol} \%)$ and $2 \mathrm{a}(0.12 \mathrm{mmol})$ in $\mathrm{CD}_{2} \mathrm{Cl}_{2}$ was monitored, followed by the addition of $\mathbf{1 a}(0.1 \mathrm{mmol})$ in to the mixture (Figure 1). The formation of zwitterion intermediate $\mathbf{A}$ in Scheme 1 , observed as a new ${ }^{31} \mathrm{P}$ NMR chemical shift, was generated at $\delta=30 \mathrm{ppm}$, and did not disappear until the reaction was finished. On the basis of the experimental results and previous related studies, a plausible transition state was proposed to explain the stereochemistry of the product (Scheme 3). We propose that after deprotonation by the basic in situ generated zwitterion, the resultant enolate form of 3-aryloxindoles might interact with the catalyst by both hydrogen bonding as well as static interaction. The presence of the $3,5-\mathrm{CF}_{3}$-substi- tuted benzene ring may block the $R e$ face of the enolate, driving the electrophile to attack from the $S i$ face.

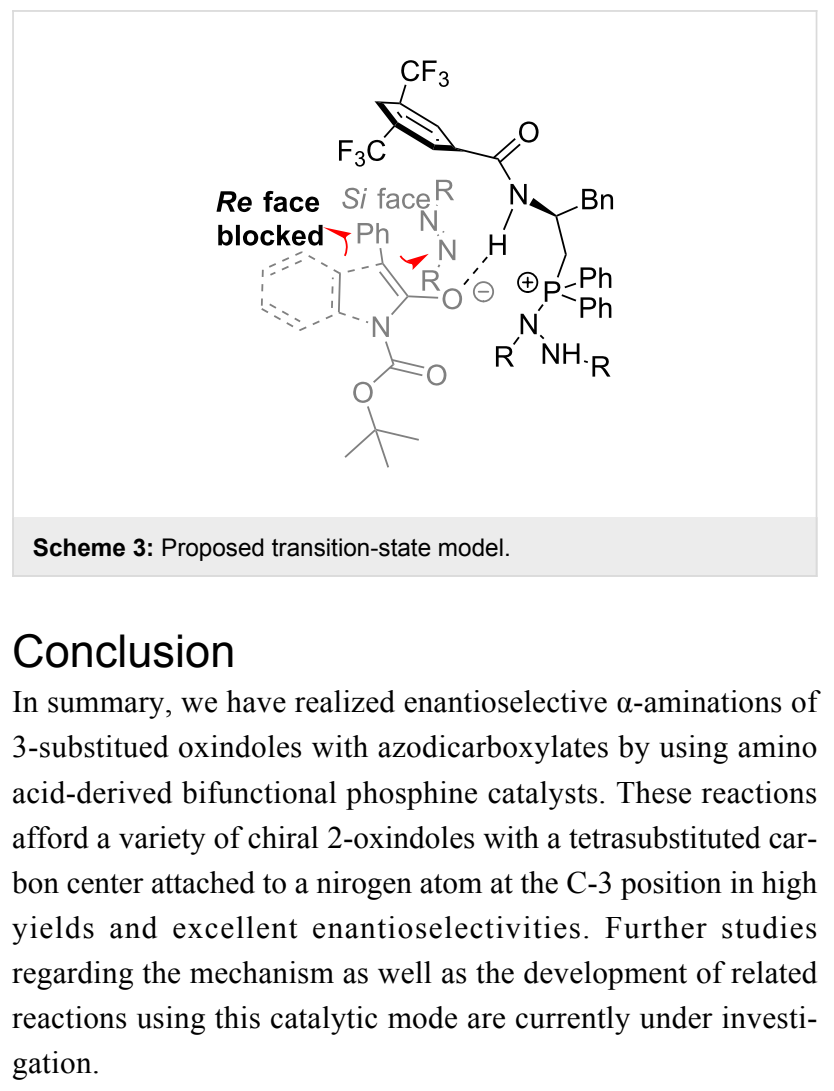

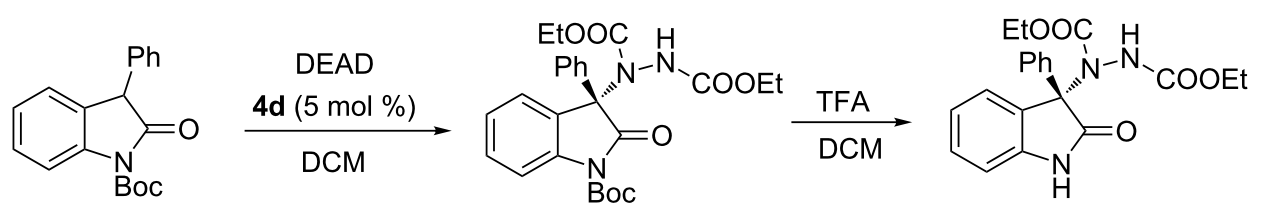

$1 a$

$1.0 \mathrm{mmol}$
5

$85 \%$ yield, $96 \%$ ee
$3 a$

$70 \%$ yield, $85 \%$ ee $96 \%$ ee after recrystallization 


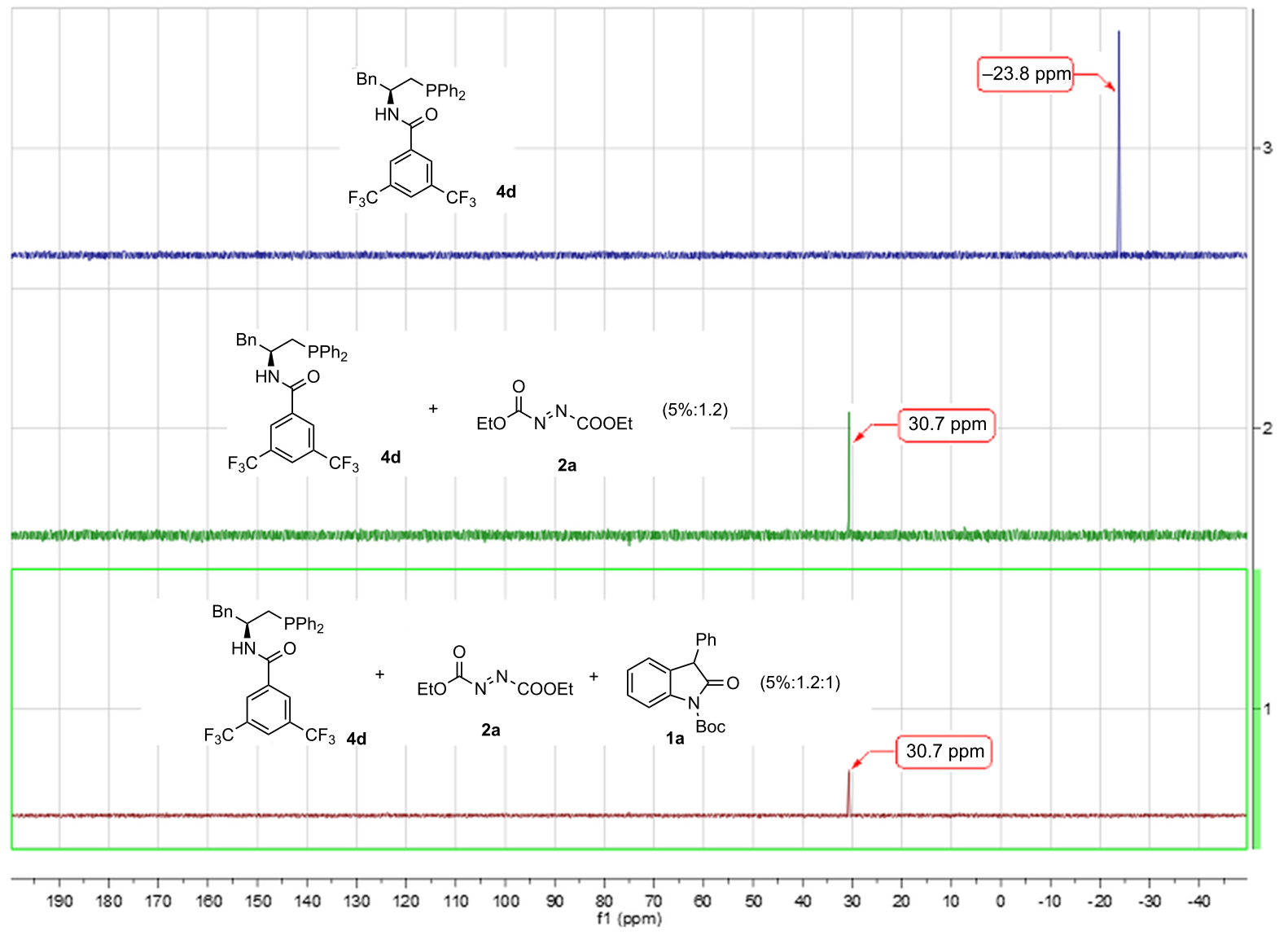

Figure 1: The ${ }^{31} \mathrm{P}$ NMR spectra research in $\mathrm{CD}_{2} \mathrm{Cl}_{2}$.

\section{Supporting Information}

\section{Supporting Information File 1}

Experimental part.

[http://www.beilstein-journals.org/bjoc/content/

supplementary/1860-5397-12-72-S1.pdf]

\section{Acknowledgements}

Financial support from the National Natural Science Foundation of China (Nos. 21572247, 21272247, 21290184) is gratefully acknowledged.

\section{References}

1. Dounay, A. B.; Overman, L. E. Chem. Rev. 2003, 103, 2945-2964. doi:10.1021/cr020039h

2. Heimgartner, H. Angew. Chem. 1991, 103, 271-297. doi:10.1002/ange.19911030306

Angew. Chem., Int. Ed. Engl. 1991, 30, 238-264. doi:10.1002/anie.199102381
3. Arend, M. Angew. Chem. 1999, 111, 3047-3049. doi:10.1002/(SICI)1521-3757(19991004)111:19<3047::AID-ANGE3047 $>3.0 . \mathrm{CO} ; 2-\mathrm{A}$

Angew. Chem., Int. Ed. 1999, 38, 2873-2874. doi:10.1002/(SICI)1521-3773(19991004)38:19<2873::AID-ANIE2873>3 .0.CO;2-P

4. Bergmeier, S. C. Tetrahedron 2000, 56, 2561-2576. doi:10.1016/S0040-4020(00)00149-6

5. Ooi, T.; Takahashi, M.; Doda, K.; Maruoka, K. J. Am. Chem. Soc. 2002, 124, 7640-7641. doi:10.1021/ja0118791

6. Suri, J. T.; Steiner, D. D.; Barbas, C. F., III. Org. Lett. 2005, 7, 3885-3888. doi:10.1021/ol0512942

7. Poulsen, T. B.; Alemparte, C.; Jorgensen, K. A. J. Am. Chem. Soc. 2005, 127, 11614-11615. doi:10.1021/ja0539847

8. Cheng, L.; Liu, L.; Wang, D.; Chen, Y.-J. Org. Lett. 2009, 11, 3874-3877. doi:10.1021/ol901405r

9. Qian, Z.-Q.; Zhou, F.; Du, T.-P.; Wang, B.-L.; Ding, M.; Zhao, X.-L.; Zhou, J. Chem. Commun. 2009, 6753-6755. doi:10.1039/B915257A

10. Zhou, F.; Ding, M.; Liu, Y.-L.; Wang, C.-H.; Ji, C.-B.; Zhang, Y.-Y.; Zhou, J. Adv. Synth. Catal. 2011, 353, 2945-2952. doi:10.1002/adsc.201100379

11. Bui, T.; Borregan, M.; Barbas, C. F., III. J. Org. Chem. 2009, 74, 8935-8938. doi:10.1021/jo902039a 
12. Bui, T.; Hernández-Torres, G.; Milite, C.; Barbas, C. F., III. Org. Lett. 2010, 12, 5696-5699. doi:10.1021/ol102493q

13. Mouri, S.; Chen, Z.; Mitsunuma, H.; Furutachi, M.; Matsunaga, S.; Shibasaki, M. J. Am. Chem. Soc. 2010, 132, 1255-1257. doi:10.1021/ja908906n

14. Yang, Z.; Wang, Z.; Bai, S.; Shen, K.; Chen, D.; Liu, X.; Lin, L.; Feng, X. Chem. - Eur. J. 2010, 16, 6632-6637. doi:10.1002/chem.201000126

15. Wang, S.-X.; Han, X.; Zhong, F.; Wang, Y.; Lu, Y. Synlett 2011, 2766-2778. doi:10.1055/s-0031-1289538

16. Zhao, Q.-Y.; Lian, Z.; Wei, Y.; Shi, M. Chem. Commun. 2012, 48, 1724-1732. doi:10.1039/C1CC15793K

17. Fan, Y. C.; Kwon, O. Chem. Commun. 2013, 49, 11588-11619. doi:10.1039/c3cc47368f

18. Wei, Y.; Shi, M. Chem. - Asian J. 2014, 9, 2720-2734. doi:10.1002/asia.201402109

19. Declerck, V.; Martinez, J.; Lamaty, F. Chem. Rev. 2009, 109, 1-48. doi:10.1021/cr068057c

20. Basavaiah, D.; Reddy, B. S.; Badsara, S. S. Chem. Rev. 2010, 110, 5447-5674. doi:10.1021/cr900291g

21. Wei, Y.; Shi, M. Chem. Rev. 2013, 113, 6659-6690. doi:10.1021/cr300192h

22. Rauhut, M.; Currier, H. Preparation of Dialkyl 2-Methylene Glutarates. U.S. Patent US3,074,999, Jan 22, 1963.

23. McClure, J. D. J. Org. Chem. 1970, 35, 3045-3048. doi:10.1021/j000834a039

24.Zhao, Q.-Y.; Pei, C.-K.; Guan, X.-Y.; Shi, M. Adv. Synth. Catal. 2011, 353, 1973-1979. doi:10.1002/adsc.201100434

25. Zhang, X.-N.; Shi, M. Eur. J. Org. Chem. 2012, 6271-6279. doi:10.1002/ejoc.201200940

26. Shi, Z.; Yu, P.; Loh, T.-P.; Zhong, G. Angew. Chem. 2012, 124, 7945-7949. doi:10.1002/ange.201203316

Angew. Chem., Int. Ed. 2012, 51, 7825-7829. doi:10.1002/anie.201203316

27. Takizawa, S.; Nguyen, T. M.-N.; Grossmann, A.; Enders, D.; Sasai, A. Angew. Chem. 2012, 124, 5519-5522. doi:10.1002/ange.201201542 Angew. Chem., Int. Ed. 2012, 51, 5423-5426. doi:10.1002/anie.201201542

28. White, D. A.; Baizer, M. M. Tetrahedron Lett. 1973, 14, 3597-3600. doi:10.1016/S0040-4039(01)86980-X

29. Trost, B. M.; Li, C.-J. J. Am. Chem. Soc. 1994, 116, 3167-3168. doi:10.1021/ja00086a074

30. Trost, B. M.; Dake, G. R. J. Org. Chem. 1997, 62, 5670-5671. doi:10.1021/jo970848e

31. Chung, Y. K.; Fu, G. C. Angew. Chem. 2009, 121, 2259-2261. doi:10.1002/ange.200805377

Angew. Chem., Int. Ed. 2009, 48, 2225-2227.

doi:10.1002/anie.200805377

32. Smith, S. W.; Fu, G. C. J. Am. Chem. Soc. 2009, 131, 14231-14233. doi:10.1021/ja9061823

33. Sun, J.; Fu, G. C. J. Am. Chem. Soc. 2010, 132, 4568-4569. doi:10.1021/ja101251d

34. Lundgren, R. J.; Wilsily, A.; Marion, N.; Ma, C.; Chung, Y. K.; Fu, G. C. Angew. Chem. 2013, 125, 2585-2588. doi:10.1002/ange.201208957 Angew. Chem. Int. Ed. 2013, 52, 2525-2528. doi:10.1002/anie.201208957

35. Wang, T.; Yao, W.; Zhong, F.; Pang, G. H.; Lu, Y. Angew. Chem. 2014, 126, 3008-3012. doi:10.1002/ange.201307757

Angew. Chem., Int. Ed. 2014, 53, 2964-2968.

doi:10.1002/anie.201307757
36. Mindal, M.; Ibrahim, A. A.; Wheeler, K. A.; Kerrigan, N. J. Org. Lett. 2010, 12, 1664-1667. doi:10.1021/ol100075m

37. Xiao, H.; Chai, Z.; Zheng, C.-W.; Yang, Y.-Q.; Liu, W.; Zhang, J.-K.; Zhao, G. Angew. Chem. 2010, 122, 4569-4572.

doi:10.1002/ange.201000446

Angew. Chem., Int. Ed. 2010, 49, 4467-4470. doi:10.1002/anie.201000446

38. Han, X.; Wang, Y.; Zhong, F.; Lu, Y. J. Am. Chem. Soc. 2011, 133, 1726-1729. doi:10.1021/ja1106282

39. Xiao, H.; Chai, Z.; Cao, D.; Wang, H.; Chen, J.; Zhao, G. Org. Biomol. Chem. 2012, 10, 3195-3201. doi:10.1039/c2ob25295c

40. Chai, Z.; Zhao, G. Catal. Sci. Technol. 2012, 2, 29-41. doi:10.1039/C1CY00347J

41. Cao, D.; Chai, Z.; Zhang, J.; Ye, Z.; Xiao, H.; Wang, H.; Chen, J.; Wu, X.; Zhao, G. Chem. Commun. 2013, 49, 5972-5974. doi:10.1039/c3cc42864h

42. Wang, H.-Y.; Zhang, K.; Zheng, C.-W.; Chai, Z.; Cao, D.-D.; Zhang, J.-X.; Zhao, G. Angew. Chem. 2015, 127, 1795-1799. doi:10.1002/ange.201409342 Angew. Chem. ,Int. Ed. 2015, 54, 1775-1779. doi:10.1002/anie.201409342

43. Mitsunobu, O.; Yamada, Y. Bull. Chem. Soc. Jpn. 1967, 40, 2380-2382. doi:10.1246/bcsj.40.2380

44.Zhong, F.; Dou, X.; Han, X.; Yao, W.; Zhu, Q.; Meng, Y.; Lu, Y. Angew. Chem. 2013, 125, 977-981. doi:10.1002/ange.201208285 Angew. Chem. ,Int. Ed. 2013, 52, 943-947.

doi:10.1002/anie.201208285 The racemic version of this reaction was reported by this group.

45. Banks, R. E.; Smart, B. E.; Tatlow, J. C. Organofluorine Chemistry: Principles and Commercial Applications; Plenum Press: New York, 1994. doi:10.1007/978-1-4899-1202-2

\section{License and Terms}

This is an Open Access article under the terms of the Creative Commons Attribution License (http://creativecommons.org/licenses/by/2.0), which permits unrestricted use, distribution, and reproduction in any medium, provided the original work is properly cited.

The license is subject to the Beilstein Journal of Organic Chemistry terms and conditions:

(http://www.beilstein-journals.org/bjoc)

The definitive version of this article is the electronic one which can be found at: doi:10.3762/bjoc. 12.72 